\title{
Democracia y alta función pública en el marco intergubernamental del Estado español
}

\author{
Democracy and senior public officials in the intergovernmental \\ framework of the Spanish State
}

\author{
Jorge Crespo-González \\ Universidad Complutense de Madrid \\ jcrespog@ucm.es
}

\section{NOTA BIOGRÁFICA}

El doctor Jorge Crespo-González es profesor de Ciencia Política y de la Administración en la Universidad Complutense de Madrid (UCM). En la actualidad además es vicedecano de asuntos económicos en la Facultad de Ciencias Políticas y Sociología de la UCM y coordinador del área de Función Pública Estratégica del Instituto Complutense de Ciencia de la Administración. Sus intereses científicos pivotan sobre la gobernanza y la gestión multinivel, función pública estratégica, función directiva y gestión de personas en las Administraciones públicas. Sus últimas publicaciones versan sobre "Coordinación intergubernamental vista por la alta función pública del Estado" (en Política y Sociedad, vol. 2, núm. 54, 2017, págs. 469-496), "Pluralismo geográfico del origen de los altos funcionarios de la Administración General del Estado" (en Mundos emergentes: cambios, conflictos, expectativas. Asociación Castellano-manchega de Sociología, 2015) y sobre Crisis y reinvención de la función pública en un escenario de gobernanza multinivel (Instituto Nacional de Administración Pública de España, 2015).

\section{RESUMEN}

Mediante un marco teórico que incluye determinantemente el enfoque intergubernamental, y a través de una metodología que incorpora un importante trabajo empírico recientemente realizado sobre altos funcionarios generalistas de la Administración del Estado, se pretende exponer algunas conclusiones centradas en cómo dichos altos funcionarios ven la realidad intergubernamental española, algunos aspectos problemáticos y propuestas de mejora. De esta manera se realiza una aportación al estudio de la democracia desde las Relaciones Intergubernamentales en España, a partir de un actor crítico, la alta función pública, de cuyas percepciones y actitudes no disponemos de demasiada evidencia empírica.

\section{PALABRAS CLAVE}

Alta Función pública; Democracia; Relaciones Intergubernamentales; Administradores civiles del Estado; Administración pública abierta; Gobernanza.

\begin{abstract}
:
In this article, the author aims to present an intergovernmental analysis on high public function of the Spanish State, based on the premise that senior officials are a critical actor for the governance of societies and they constitute too a good instrument to know basic aspects of the democracy of states. The research methodology has incorporated an empirical work applied on the Cuerpo Superior de Administradores Civiles del Estado, the only collective of the Spanish General State Administration with a generalist dimension who participates in the formulation and implementation of intergovernmental
\end{abstract}


policies. Besides that, research offers interesting data about geographical origin of the senior public officials in Spain; the need of promoting multilevel public career; and the roles of these officials on the complex Spanish State.

\title{
KEYWORDS
}

Public officials; Spain; Intergovernmental Relations; National Administration; Senior Public Service; Federalism; Governance.

\begin{abstract}
SUMARIO
AGRADECIMIENTOS. INTRODUCCIÓN. 1. ALTA FUNCIÓN PÚBLICA Y ANÁLISIS INTERGUBERNAMENTAL. 1.1. FUNCIÓN PÚBLICA Y ALTA FUNCIÓN PÚBLICA. 1.2. ESTUDIOS DE LA FUNCIÓN PÚBLICA DESDE LA PERSPECTIVA INTERGUBERNAMENTAL. 1.3. QUÉ APORTAN LOS ESTUDIOS DESDE LAS RIG EN EL MARCO DE LA CIENCIA POLÍTICA Y DE LAADMINISTRACIÓN ESPAÑOLA. 2. ASPECTOS INTERGUBERNAMENTALES CON IMPACTO REPRESENTATIVO RESPECTO DE LAALTA FUNCIÓN PÚBLICA DEL ESTADO. 2.1. PARTICIPACIÓN Y ROLES INTERGUBERNAMENTALES DE LOS ACE EN LAS POLÍTICAS PÚBLICAS MULTINIVEL EN ESPAÑA. 2.2. SOBRE E INFRARREPRESENTACIÓN AUTONÓMICA EN LA ALTA FUNCIÓN PÚBLICA DEL ESTADO. EL CASO DE LOS ACE. 2.3. AGENDA PENDIENTE PARA MEJORAR LAS CAPACIDADES INTERGUBERNAMENTALES DE LA ALTA FUNCIÓN PÚBLICA. 3. CONCLUSIONES.
\end{abstract}

\section{AGRADECIMIENTOS}

El autor quiere reconocer expresamente la ayuda prestada para la realización del trabajo empírico por diversas instituciones, particularmente el Instituto Nacional de Administración Pública de España y la Asociación profesional del Cuerpo Superior de Administradores Civiles del Estado. También está en deuda con los 110 altos funcionarios encuestados y/o entrevistados por su imprescindible contribución. Recuerda asimismo los muchos comentarios estimulantes recibidos desde el Instituto Complutense de Ciencia de la Administración (ICCA) y el Departamento de Ciencia Política y de la Administración (UCM). Y, finalmente, no quiere dejar pasar la ocasión de agradecer las valiosas sugerencias proporcionadas por los revisores anónimos, así como las obtenidas en la presentación de una versión provisional de este trabajo en el XIII Congreso de la Asociación Española de Ciencia Política y de la Administración (Santiago de Compostela, octubre de 2017).

\section{INTRODUCCIÓN}

El presente análisis da cuenta de una investigación más amplia en que se analizó el rol intergubernamental de la alta función pública española, tomando como ejemplo a efectos de extracción de información el Cuerpo Superior de Administradores Civiles del Estado (ACE en lo sucesivo), único de los colectivos al servicio de la Administración general del Estado (AGE) español con carácter generalista y con funciones predirectivas y directivas directamente implicado en actividades con proyección multinivel ${ }^{1}$.

La importancia de la alta función pública a efectos intergubernamentales ha sido puesta de relieve por la literatura científica estadounidense en la primera mitad del siglo xx (McCULLOCH 1965; o WEIDNER 1960). Más recientemente, otros autores han enfatizado sus funciones de acceso al centro político (AGRANOFF 1991), o las capacidades adhesivas y lubricantes para el sistema político (PARRY 2012 y 2004), pero la realidad es que los estudios de base empírica son la excepción y los que se focalizan en la gobernanza multinivel y las actitudes y percepciones de la función pública prácticamente inexistentes (CRESPO 2017).

El trabajo empírico incorporó la respuesta a un cuestionario de 102 de sus miembros y la realización de múltiples entrevistas a académicos de la talla de Sabino Cassese, Manuel Arenilla o Mariano Baena del Alcázar. La fase de obtención de datos finalizó en el año 2016 y debemos agradecer la colaboración de la Asociación Profesional del Cuerpo Superior de Administradores Civiles del Estado y del Instituto Nacional de Administración Pública de España. Cuando en este estudio se aluda a la información extraída de las entrevistas, se citará incluyendo el término «ENTREV.» junto al apellido del entrevistado (por ejemplo, ENTREV. CASSESE). 
Como excepción reseñable, caben citarse los trabajos realizados en el marco comunitario europeo que enfatizan la coordinación para la cohesión en el sector público del futuro (COCOPS, según abreviatura en lengua inglesa), en que hay una sección dedicada a las percepciones y experiencias sobre la reforma del sector público en España (ALONSO y CLIFTON, 2013), donde se trata sobre todo cómo ven el impacto de la Nueva Gestión Pública los altos funcionarios. Otro estudio relevante y reciente, de carácter comparativo, es el realizado por KURERUS y RODE (2016), centrado en el acceso y gestión de los altos funcionarios en las Administraciones centrales de 28 países europeos, pero sin dar datos de interés sobre el perfil intergubernamental de los mismos.

En este contexto, el presente trabajo pretende analizar y explicar qué posición y visión tiene la alta función pública del Estado, a través de los ACE, respecto de la participación de los burócratas españoles en la gobernanza española, en términos funcionales e institucionales. Aspecto de máximo interés, habida cuenta del contexto en que opera España (fuerte descentralización política interna y gran complejidad externa), así como del replanteamiento actual de aspectos democráticos vinculados al funcionamiento del Estado, su Administración y la política. El autor asume la importancia de considerar la Administración pública como un espejo de la sociedad a la que sirve, y en la que, por lo tanto, desde una perspectiva institucional y democrática, debería incluirse la diversidad y complejidad de la ciudadanía. El interés del asunto no es menor, dada la relevancia de la alta función pública para la generación de la cultura político-administrativa, para la conformación del liderazgo político y por la constatación de que de la alta función pública se nutren las élites públicas y privadas del país (ARENILLA, 2010 y 2014). Por ello, después de justificar el interés de analizar la alta función pública desde una perspectiva de RIG, y contando con la apoyatura del trabajo empírico desarrollado, el estudio se plantea varios objetivos específicos: en qué radica la importancia del trabajo multinivel de la alta función pública española; los roles que desempeñan los altos funcionarios de carácter generalista; qué impacto tiene la procedencia geográfica de la alta función pública y, si el mismo fuera considerado como negativo o disfuncional, qué acciones cabría emprender para mitigar ese sesgo territorial en el ingreso a la misma. A este fin, se subraya un elenco de actividades que permitiría reducir el sesgo representativo de índole territorial de la alta función pública de la AGE, destacándose el redescubrimiento de la movilidad interadministrativa.

En el desarrollo del estudio se comenzará por definir qué se considera como alta función pública, para posteriormente realizar un breve análisis sobre los estudios que conectan la misma con los aspectos intergubernamentales o multinivel, con el fin de destacar aquellos aspectos en que la alta función pública influye sobre la gobernanza española. A continuación, se plantearán algunos sesgos representativos que afectan a la alta función pública del Estado, destacando los territoriales y la agenda pendiente desde una perspectiva práctica para mitigarlos. Y finalmente se extraerán algunas conclusiones, no siendo la menor la necesidad de reforzar los elementos representativos de la alta función pública del Estado como contribución para mejorar la calidad de la democracia en España.

\section{ALTA FUNCIÓN PÚBLICA Y ANÁLISIS INTERGUBERNAMENTAL}

Antes de concretar algunos de los sesgos a efectos de representatividad, es imprescindible definir qué se entiende por función pública, así como el estado del conocimiento sobre la misma desde una perspectiva intergubernamental.

\subsection{Función pública y alta función pública}

El concepto de «función pública» tiene una dimensión funcional y otra personal. La primera se desprende del sustantivo «función», y alude a la actividad a través de la cual los miembros de una sociedad articulan procedimientos para garantizar la cohesión y reproducción social, así como para garantizar las relaciones y el control entre los distintos poderes del Estado, tanto entre sí como respecto de los ciudadanos. La segunda, que es la que más interesa a efectos de este trabajo, alude al elemento humano que, dependiendo de la sociedad y al servicio de ella, labora en el marco de la Administración. Esta concepción de la función pública en tanto colectivo humano debe matizarse, pues si bien todos los recursos humanos o empleados públicos tienen alguna conexión con el poder público por el mero hecho de trabajar en la Administración, lo cierto es que algunos de ellos, individualmente u organizados en colectivos o cuerpos, son los que ejercen el poder 
administrativo al máximo nivel, y es en ellos en los que se centra este trabajo, singularmente en el Cuerpo de Administradores Civiles del Estado (ACE), los antiguos Técnicos de Administración Civil (TAC)

La alta función pública históricamente se ha identificado con una fuerte cualificación y profesionalización, así como con una posición elevada en el sistema político-administrativo. A efectos operativos, y siguiendo la tradición de los estudios sobre burocracia en España, se ha optado por identificarla con el segmento de funcionarios que forman parte de los cuerpos superiores (que exigen titulación universitaria de carácter superior en el acceso) y que desempeñan funciones estratégicas en la AGE (BAENA, 1988), ya sea por su participación en la formulación de políticas y/o implantación de las mismas, como por su posición central en el entramado de interacciones que vincula a la política con la sociedad a través de la Administración. Entre los colectivos que forman parte de la alta función pública española de la AGE destaca el Cuerpo Superior de Administradores Civiles del Estado, cuyas funciones tienen un claro compromiso multinivel, y sobre el que se ha aplicado el trabajo empírico en esta investigación.

\subsection{Estudios de la función pública desde la perspectiva intergubernamental}

Respecto del estado del conocimiento científico sobre la función pública en España cabe apreciar que se cifran por cientos las monografías y artículos de revistas especializadas en que se trata el asunto, aunque sin embargo el tratamiento de la misma desde la Ciencia Política y de la Administración no es tan abundante y desde una perspectiva intergubernamental es escasa y fragmentaria, alcanzando cuando se trata el asunto a describir algunos aspectos del complejo entorno que afecta al personal público, pero sin darle (salvo excepciones) un desarrollo propio y profundo en términos institucionales ni de capacidades que debe el personal público incorporar.

El análisis de la bibliografía seleccionada constata que los estudios que dan una relevancia macro o institucional a la función pública son la excepción y que, sin embargo, predominan (como en el universo del que son muestra) los realizados bajo parámetros jurídicos o de mera gestión de personal. Lo que permite afirmar que, salvo las excepciones a que después se hará mención, los estudios en el pasado inmediato y en la actualidad sobre empleo público en España se centran en el análisis de los modelos comparados, el acceso y la selección, la formación, la carrera, el sistema retributivo, la buena administración y ética, el desempeño, eficacia y eficiencia, la gestión por competencias, el impacto del Estatuto Básico del Empleado Público (EBEP), la dirección pública profesional y el impacto de la crisis económica (CRESPO 2015b, 34). $Y$ que ello pone de manifiesto la necesidad de nuevos estudios multidisciplinares en que, sin menosprecio de los aspectos instrumentales, se reconozca y ponga en valor la importancia de la función pública en tanto elemento estructuralmente necesario para la sociedad, arraigado en las instituciones del Estado, y que por añadidura se acompañen del impacto que la gobernanza actual imprime a nuestra democracia (CRESPO, ibídem: 33).

En este contexto, la aportación desde las Relaciones Intergubernamentales (RIG) es especialmente interesante y conecta naturalmente con las preocupaciones de la gobernanza multinivel en que la gestión de los asuntos públicos se sustancia en nuestros días.

Como es conocido, las RIG constituyen un enfoque analítico que nace en EEUU en los años 30 del pasado siglo y que hace énfasis en el estudio del funcionamiento de los Estados a partir de cómo se sustancian las relaciones entre unidades de gobierno y administración de los distintos niveles de gobierno, dando por lo tanto especial relevancia no tanto a las categorías más o menos rígidas del Derecho Público como a aspectos dinámicos tales como la interacción entre actores públicos en un contexto de participación multidireccional y fluida, y en que cobran especial interés las reflexiones y técnicas para comprender y encarar el conflicto, la cooperación, la coordinación y la negociación.

Las RIG incorporan unas pautas de análisis en las que el factor humano tiene una consideración especial, como se desprende de la obra de uno de los clásicos (ANDERSON, 1960), que además de expresar que la gestión y administración intergubernamental debe formularse necesariamente en términos de relaciones y conductas humanas, concreta el atractivo de estudiar aspectos como el número y variedad de funcionarios públicos intervinientes, la intensidad y regularidad de los contactos entre ellos, o la importancia de las acciones y actitudes de los funcionarios. El interés de estos aspectos ha sido realzado por la literatura científica; por ejemplo, $\mathrm{CHO}$ destacó la importancia de las actitudes y acciones de los oficiales públicos, pues cómo ven y perciben ellos el sistema político en acción es una dimensión importante en términos intergubernamentales, aunque también lamenta que no haya demasiada investigación empírica y sean escasos 
los hallazgos (CHO 2007, 51). Sin duda, este autor tenía muy presente que desde los estudios empíricos estadounidenses de mediados del siglo pasado, impulsados desde la Universidad de Minnesota, no ha habido aportaciones relevantes con base empírica.

La estela de ANDERSON ha sido seguida por otros autores, entre los que cabe destacar a los siguientes, sin ánimo de exhaustividad, por ser aquellos que han generado las aportaciones sobre las que pivota el debate a nivel internacional.

Por una parte, WRIGHT (1978 y 1997), quien además incorpora un sugestivo esquema para clasificar a los Estados de acuerdo con la tipología de relaciones que se producen entre los diferentes niveles de gobierno, definiendo básicamente 3 posibilidades: modelo inclusivo, separado y superpuesto. Por otra, AGRANOFF, cuya obra es rica en alusiones a la importancia del empleo público en el marco de las RIG (sin olvidar que en el ámbito norteamericano se incluye dentro del mismo al personal público procedente de designación política y nombramiento) y que asignó a los empleados públicos la importante función de acceder al centro político mediante sus grupos de especialistas funcionales, las élites locales y los funcionarios de nivel nacional. Asimismo, incluyó como enfoque emergente de investigación la conducta de los participantes en las RIG, y expresamente a los empleados públicos (AGRANOFF, 1991: 26).

Otro estudioso de la importancia de la función pública a nivel intergubernamental es PARRY, quien analiza el Civil Service británico y plantea una muy interesante conclusión: que la alta función pública es un factor que puede potencialmente favorecer unas buenas RIG en Reino Unido, pues incorpora funciones lubricantes y adhesivas sobre el sistema político-administrativo (PARRY 2012), aspecto al que sin duda podría añadirse el argumento proporcionado por STEIN (1984) al apuntar que las RIG y el gobierno mejorarían si se impulsaran carreras multinivel para los empleados públicos. Finalmente, y en la línea de ponderar la importancia de la función pública cabe citar el planteamiento de VERKUIL (2015), que la visualiza como una infraestructura humana que permite a las sociedades reaccionar ante situaciones críticas con una mejor y mayor resiliencia.

Los estudios de la función pública desde una perspectiva intergubernamental en España son escasísimos y se han ocupado preferentemente del reparto y negociación de competencias, del marco general de cooperación y coordinación, la futura forma del Estado español, la financiación autonómica o el análisis multinivel de alguna política pública concreta (véase las temáticas sobre las que versan los estudios intergubernamentales en España en COLINO 2012). Merece su consulta, por ejemplo, a tales efectos los elaborados por ALDA (2010) y SANIGER (2005), sobre el cambio político, evolución y disfunciones de las RIG en España, o sus dimensiones institucionales (ALDA, CICUÉNDEZ y RAMOS, 2006). Pero, como se ha señalado, son excepción los que se centran en los actores críticos sustantivando entre éstos a la función pública, lo que no impide que cada vez son más los autores que, de manera directa o indirecta, plantean la necesidad de que los análisis intergubernamentales incorporen la función pública como elemento explicativo del funcionamiento del Estado (por ejemplo, GARCÍA MORALES, 2006: 10), o por la importancia que el modelo relacional entre políticos y funcionarios tiene para la configuración y adopción de decisiones políticas, así como para su ulterior implantación (ARENILLA, 2010: 53-54).

Asumiendo lo anterior, en los últimos años se constata en España un incremento de la preocupación sobre los actores en general, y sobre la alta función pública en particular, junto con el respaldo de análisis empíricos, entre estudiosos españoles. Primeramente, es de interés el trabajo elaborado por RUANO et al. (2014) que analiza el ciclo selectivo del personal de la AGE, centrándose en aquellos cuerpos cuyos procesos selectivos son gestionados desde el Instituto Nacional de Administración Pública (INAP), entre los que se halla el de ACE; de dicho trabajo merecen retenerse especialmente los datos obtenidos respecto de la procedencia territorial de los ACE, que junto con los proporcionados por ALVAREZ (1980), nos permitieron extraer conclusiones sobre la inalterable sobre-representación de Madrid y las comunidades mesetarias, frente a las periféricas, en un estudio que versó sobre el pluralismo geográfico del origen de los altos funcionarios de la AGE (CRESPO, 2015c). En segundo lugar, merece mención la obra publicada por DE LA PEÑA et al. (2015), sobre la dinámica de funcionamiento y los valores y la percepción de los agentes políticos y técnicos en las conferencias sectoriales entre 2001-2012, pues aunque el análisis se focalice en la participación en un instrumento de cooperación (las conferencias sectoriales), se otorga cierto protagonismo a la alta función pública. Y finalmente, debe destacarse muy singularmente la obra coordinada por ARBÓs (2009), sobre la posición de los actores en las relaciones intergubernamentales en el Estado autonómico, la cual alude con frecuencia a las aportaciones que los funcionarios hacen a las mismas, por su participación esencialmente en la preparación técnica de las reuniones de los órganos de cooperación. Asimismo, dicha obra 
incorpora varias páginas sobre la capacitación de los empleados del Estado para asumir favorablemente y con visos de éxito las nuevas funciones que el entorno intergubernamental les depara.

\subsection{Qué aportan los estudios desde las RIG en el marco de la Ciencia Política y de la Administración española}

Los estudios de la función pública desde las RIG se combinan bien con los establecidos desde la perspectiva politológica de la Ciencia de la Administración española. Tal como destaca ARENILLA (2016), hay una fértil línea de investigación destinada al estudio de la burocracia y las élites administrativas (por ejemplo, BAENA, 1999; PARRADO, 1996; ROMÁN, 1997; RUANO et al., 2014), de donde se extraen valiosas conclusiones respecto de su posición en el sistema y estructura de poder españoles y se establece un nexo necesario entre sociedad, Estado y democracia a través de la alta función pública.

No en vano, se estima que la alta función pública forma parte del conjunto decisional político (ARENILLA, 2010), tanto por las funciones que desempeña, como por las importantes relaciones de intercambio, influencia mutua y solapamiento entre los colectivos de altos funcionarios y políticos. De manera que se mantiene que analizar la alta función pública dista de ser un mero ejercicio técnico por su posición (funcional y/o personal) en el entramado o grupo de poder que adopta las decisiones conformadoras, lo que además de justificar su estudio implica su conexión con importantes aspectos institucionales, y particularmente las relaciones en tanto nexo conector indispensable entre ciudadanía, democracia, Administración y Estado.

$\mathrm{Si}$, como indican las evidencias, lo anterior es cierto, analizar empíricamente el rol de los directivos públicos y altos funcionarios en un sistema político incorpora un interés hasta ahora poco estudiado, y debe considerarse su conexión con aspectos institucionales y democráticos de base, de lo que se derivan importantes interrogantes; un ejemplo de los mismos sería si el acceso al empleo público a través de esos colectivos no prefigura directa o indirectamente la participación en las decisiones políticas conformadoras, y si esto fuera así, entonces se impondría la necesidad de reflexionar sobre los sesgos asociados al acceso desde una perspectiva socio-económica pero también desde una perspectiva territorial, máxime en un Estado, como el español, con fuertes sensibilidades respecto del rol e identidad de la AGE. Lo anterior hace emerger una cuestión central, de gran interés político y científico en palabras de ARENILLA (2014: 28): la representatividad de la función pública respecto de la sociedad a la que sirve, impactada por sesgos territoriales, socio-económicos y educativos entre otros.

\section{ASPECTOS INTERGUBERNAMENTALES CON IMPACTO REPRESENTATIVO RESPECTO DE LA ALTA FUNCIÓN PÚBLICA DEL ESTADO}

El debate sobre la alta función pública a efectos institucionales no puede omitir ejes de reflexión como los siguientes: su grado de politización, su apertura a los ciudadanos y orientación a las competencias profesionales y, finalmente, su carácter representativo de la sociedad. Respecto de este último vector, y centrado en los aspectos intergubernamentales, esta parte pretende responder a las preguntas siguientes: ¿son importantes las funciones multinivel de la alta función pública española?, ¿cuáles son los roles que despliegan los ACE como exponentes de esa alta función pública? ¿qué importancia tiene la procedencia geográfica de la alta función pública en su desempeño? ¿qué pasos podrían impulsarse para mitigar los efectos disfuncionales de una procedencia geográfica de la alta función pública desequilibrada?

\subsection{Participación y roles intergubernamentales de los ACE en las políticas públicas multinivel en España}

En el trabajo empírico, se solicitó información a los ACE sobre la relevancia de sus funciones intergubernamentales y los principales roles que asumían respecto de las políticas multinivel en España.

Primeramente, y antes de caracterizar las principales funciones de los ACE desde el punto de vista intergubernamental, se les interrogó por su percepción del grado en que dicho colectivo colabora funcionalmente, junto a los políticos, en la confección de las decisiones públicas y, asimismo, en la implantación de las decisiones públicas con impacto autonómico. Con lo primero, se trataba de constatar la medida en que 
Ios ACE intervienen en lo que el profesor BAENA (1988: 441) denomina «veto decisorio» de la burocracia; y con lo segundo, en lo que el mismo profesor califica como «veto paralizante» o ejecutorio.

Respecto de su participación en la confección de decisiones, los datos plantean que los ACE respaldan la afirmación de que los mismos colaboran decisivamente con los políticos en la confección de las decisiones públicas con incidencia autonómica $(67,7 \%$ de acuerdo y totalmente de acuerdo, frente al 7,9\% en desacuerdo y totalmente en desacuerdo) $)^{2}$.

Algo similar puede establecerse respecto de la afirmación relativa a que las funciones de los ACE son decisivamente necesarias para implantar decisiones públicas del Estado con repercusión autonómica $(78,4 \%$, vs. $9,8 \%)$.

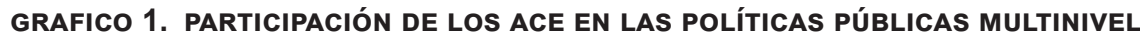

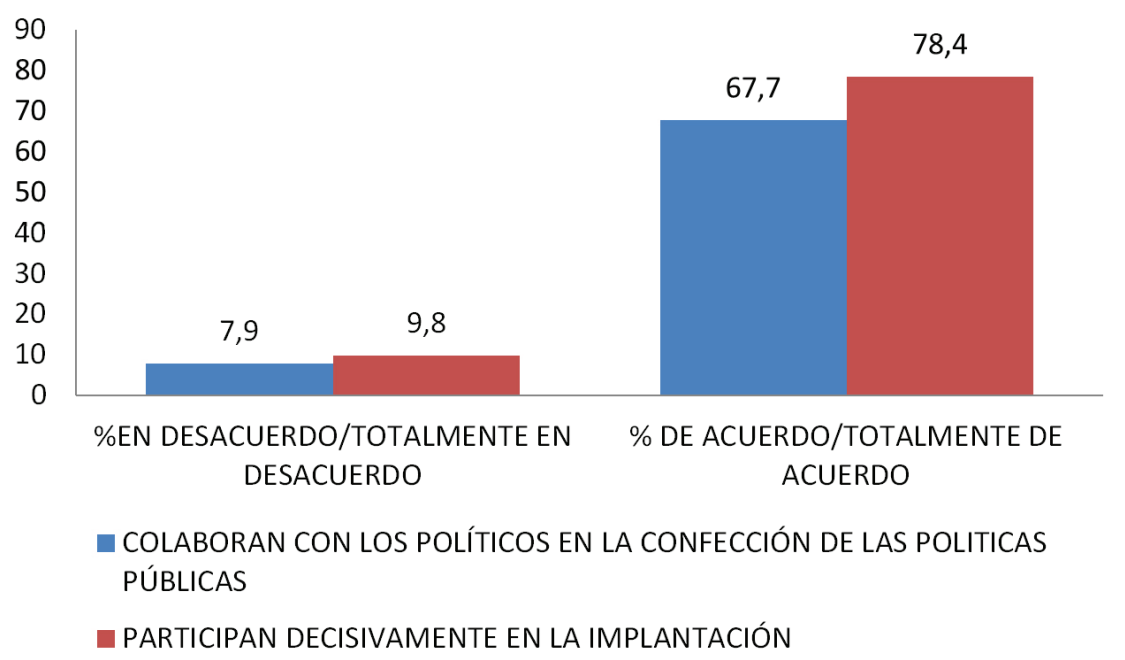

Fuente: elaboración propia.

Una vez destacada la importancia de las funciones de los ACE con implicaciones autonómicas, el foco se puso en los roles que, de acuerdo con su experiencia, pueden adoptar los altos funcionarios en ejercicio en un escenario multinivel. La pregunta se inspiraba en el estudio clásico realizado por ABERBACH, PUTNAM y ROCKMAN (1981) que mediante un examen comparado entre seis países (EEUU, Reino Unido, Alemania, Países Bajos, Francia e Italia), puso en evidencia, entre otros aspectos, los diferentes roles político-administrativos que intervienen en los procesos decisorios. La aplicación de los diversos roles confeccionados en la investigación de ABERBACH y al. al colectivo de los ACE concretó que los principales con los que se identifican son los siguientes: el de técnico que ejerce una competencia $(89,2 \%)$; el de garante de la imparcialidad del Estado (87,3\%); el de ideadores y formuladores de nuevos programas $(71,6 \%)$ y el de expertos legales $(68,6 \%)$. Sin embargo, los que cuentan con menor apoyo son aquellos que implican una adopción partidaria o de interés a instancia de parte, quedando claramente establecido su falta de apoyo, por ejemplo, al rol partidista (2,9\%), al de patrocinio de intereses $(5,9 \%)$ y al de bróker, que negocia intereses diferentes $(15,7 \%)$. Lo que significa que el colectivo estudiado interpreta sus expectativas de actuación en términos ligados a la competencia técnica y jurídica, pero también respecto de los valores básicos del servicio público (neutralidad e imparcialidad), sin despreciar aspectos creativos (rol de ideadores y formuladores) los que facilitan la interacción (facilitadores y mediadores). La significativa, aunque no determinante ni apabullante, relevancia otorgada a los roles relacionados con la interacción en tanto facilitadores y mediadores entre las demandas ciudadanas, territoriales y otros actores críticos como instrumento de canalización hacia el poder político, contrasta con una visión conformista de carácter tecnocrática, fundada en una visión de sus responsabilidades en torno a la competencia técnica y la garantía de la imparcialidad del Estado.

2 En lo sucesivo para favorecer la comparación se opta por presentar los datos agrupados de las respuestas «De acuerdo y totalmente de acuerdo» vs. «En desacuerdo y totalmente en desacuerdo», no citándose las indiferentes. 
GAPP. Nueva Época - N.o 19, mayo 2018 - ISSN: 1989-8991 - DOI: 10.24965/gapp.v0i19.10512 - [Págs. 17-33]

Democracia y alta función pública en el marco intergubernamental del Estado español Jorge Crespo-González

TABLA 1. ROLES QUE SE CONCRETAN EN EL EJERCICIO PROFESIONAL DE LOS ACE

\begin{tabular}{lr}
\hline ROLES & $\%$ \\
\hline El rol técnico de expertos que ejercen una competencia & 89,2 \\
\hline El rol de patrocinio de intereses & 5,9 \\
\hline El rol de agente (bróker), que negocia intereses diferentes & 15,7 \\
\hline El rol de facilitadores, que median entre intereses de grupos & 52 \\
\hline El rol partidista, que defiende una posición política bien clara & 2,9 \\
\hline El rol de ideadores y formuladores de nuevos programas & 71,6 \\
\hline El rol de garante de la imparcialidad del Estado & 87,3 \\
\hline El rol de expertos legales & 68,6 \\
\hline El rol de defensor cívico que protege los derechos de la colectividad & 42,2 \\
\hline Otro & 1,0 \\
\hline
\end{tabular}

Elaboración propia a partir de ABERBACH, PUTNAM y ROCKMAN (1981).

Llegados a este punto, y como anticipo de la importancia que se otorga en la investigación a la procedencia geográfica de los altos funcionarios, se indagó sobre si dicho origen geográfico podía tener influencia en el ejercicio de los diversos roles expresados anteriormente. Los resultados sugieren que los ACE dan una importancia relativamente baja a la relación existente entre origen del miembro y la mejoría en la función o rol desempeñado. En efecto, en ningún caso supera el $50 \%$ aquellos que indican que esa relación podría mejorar su rol de garantes de la imparcialidad $(45,1 \%)$, de facilitadores y mediación entre grupos $(41,2 \%)$ y de ideación y formulación de políticas $(33,3 \%)$, mientras que son muchos menos los que plantean que dicha relación mejoraría el ejercicio del rol partidista $(2,9 \%)$, de patrocinio de intereses $(10,6 \%)$, así como el de técnico ligado a la experticia $(17,6)$ y a las leyes $(13,7 \%)$. Estos resultados son coherentes con la escasa simpatía que, a tenor de las respuestas abiertas, suscita asociar criterios geográficos a las pruebas de selección en los altos cuerpos de la función pública del Estado, y que además se ve confirmada con los resultados extraídos de las entrevistas en profundidad.

TABLA 2. ¿FACILITA EL ORIGEN GEOGRÁFICO EL EJERCICIO DE LOS ROLES CON DIMENSIÓN INTERGUBERNAMENTAL?

\begin{tabular}{lcc}
\hline ROLES & $\begin{array}{c}\text { \% Origen } \\
\text { geográfico } \\
\text { facilita... }\end{array}$ & $\begin{array}{c}\% \text { General } \\
\text { anterior }\end{array}$ \\
\hline El rol técnico de expertos que ejercen una competencia & 17,6 & 89,2 \\
\hline El rol de patrocinio de intereses & 10,8 & 5,9 \\
\hline El rol de agente (bróker), que negocia intereses diferentes & 17,6 & 15,7 \\
\hline El rol de facilitadores, que median entre intereses de grupos & 41,2 & 52,0 \\
\hline El rol partidista, que defiende una posición política bien clara & 2,9 & 2,9 \\
\hline El rol de ideadores y formuladores de nuevos programas & 33,3 & 71,6 \\
\hline El rol de garante de la imparcialidad del Estado & 45,1 & 87,3 \\
\hline El rol de expertos legales & 13,7 & 68,6 \\
\hline El rol de defensor cívico que protege los derechos de la colectividad & 26,5 & 42,2 \\
\hline Otro & 21,6 & 1,0 \\
\hline
\end{tabular}

Elaboración propia a partir de ABERBACH, PUTNAM y ROCKMAN (1981). 
Y finalmente, y buscando concretar los ámbitos en que se sustancia la participación intergubernamental de las funciones que desarrollan los ACE, se les solicitó que señalasen las opciones pertinentes de acuerdo con la experiencia de cada cual en el cuerpo. Se buscaba destacar el espacio orgánico y funcional de cooperación existente en nuestro país y la contribución a su funcionamiento por parte de los ACE. Las respuestas sugieren el compromiso del cuerpo con la gobernanza multinivel de España. Su participación en asuntos intergubernamentales o con contenido intergubernamental se muestra especialmente clara e influyente sobre el colectivo de los políticos, ya sea en la elaboración de respuestas a preguntas parlamentarias $(63,4 \%)$, o en la co-elaboración de actos normativos $(61,4 \%)$, o directamente en tareas de asesoramiento $(43,6 \%)$; también es muy relevante en lo relativo a la gestión de las políticas públicas, pues un porcentaje significativo dice haber participado en la implantación de las mismas con impacto intergubernamental $(57,4 \%)$, o en su evaluación $(41,6 \%)$. Aunque de manera no mayoritaria, también es muy frecuente su participación en las conferencias sectoriales $(46,5 \%)$ y en las conferencias bilaterales (35,6\%). En las respuestas a la pregunta abierta dentro del cuestionario se proporcionó una de las claves para comprender la importante influencia de los altos cuerpos sobre los políticos en asuntos intergubernamentales: «Los funcionarios deberíamos quedarnos en un nivel técnico y neutral. El problema es que, en ocasiones, la preparación de nuestros políticos es tan nefasta que la aportación de los funcionarios se convierte en más importante y vinculante. Eso, evidentemente, varía según el gobierno y la preparación de sus miembros».

TABLA 3. DIMENSIONES EN QUE SE SUSTANCIA LA PARTICIPACIÓN INTERGUBERNAMENTAL DE LOS ACE

\begin{tabular}{lc}
\hline DIMENSIONES & $\%$ \\
\hline He participado en conferencias sectoriales o en sus órganos preparatorios & 46,5 \\
\hline He participado en conferencias bilaterales o en sus órganos preparatorios & 35,6 \\
\hline He participado en la redacción de respuestas parlamentarias con contenido intergubernamental & 63,4 \\
\hline He asesorado a órganos políticos sobre asuntos intergubernamentales & 43,6 \\
\hline He participado en la elaboración de actos normativos con impacto intergubernamental & 61,4 \\
\hline He participado en la implantación de normativa con impacto intergubernamental & 57,4 \\
\hline He participado en la evaluación de políticas públicas intergubernamentales & 41,6 \\
\hline Otro & 13,9 \\
\hline
\end{tabular}

Fuente: elaboración propia.

\subsection{Sobre e infrarrepresentación autonómica en la alta función pública del Estado. El caso de los ACE}

Como ya se ha señalado, el análisis de la procedencia geográfica de los miembros de la alta función pública de la AGE tiene mucho interés, pues es un aspecto que puede facilitar las relaciones (formales e informales) entre niveles de gobierno, y modificar las visiones y las actitudes de los ciudadanos ante ella, lo que también genera legitimidad e identificación con la actuación pública.

Ya en otro estudio (CRESPO, 2015c) se demostró que respecto de la procedencia geográfica de los TAC/ACE en perspectiva histórica cabe destacar la persistencia de la fuerte sobrerrepresentación histórica de la región madrileña, así como de Castilla y León, y la mejoría en los últimos años en la representación de Galicia, Asturias y Aragón. Sin embargo, sorprendían entre las infrarrepresentadas Cataluña, que partiendo de una base débil en los años 60 y 70, no solo no ha mejorado sino lo contrario a lo largo del tiempo. Lo mismo puede decirse de Extremadura, Navarra y Canarias.

A esos efectos, asombra que a pesar de los años transcurridos desde que se realizaron los primeros estudios sobre el origen geográfico de los funcionarios de la AGE (ALVAREZ, 1980), y del alto grado de descentralización política alcanzado por el Estado español, no se haya conseguido reducir o minorar significativamente el sesgo, fundamentalmente madrileño y mesetario, de la procedencia geográfica de los altos funcionarios, como demuestra el caso de los ACE. Indagar sobre este aspecto, sus causas y posibles soluciones, nos parece esencial y por ello en el trabajo empírico se incorporaron unas preguntas para intentar aclarar los motivos de la sobrerrepresentación e infrarrepresentación de los originarios de unas CCAA sobre otras, si se considera relevante el fenómeno y por qué, y alguna posible solución al asunto. 
Respecto de las causas de la sobre-representación, se destacó en la encuesta la importancia de la ubicación geográfica de los preparadores y la reproducción profesional entre padres e hijos por tradición familiar. Esos aspectos fueron respaldados por las entrevistas, donde también se aludió a la necesidad de mejorar las condiciones laborales de los ACE en el territorio, la cultura pública de la Comunidad Autónoma y la existencia de sectores productivos alternativos. Curiosamente, no se otorga gran importancia a tener cerca centros formativos de educación superior que oferten titulaciones apropiadas, aspecto históricamente realzado por la literatura. En lo relativo a la infra-representación, la encuesta plantea un apoyo mayoritario a la opción que subrayaba la importancia de disponer de una Administración pública autonómica amplia y diversificada que oferta puestos similares a los ACE, y un apoyo muy significativo a la de no disponer de preparadores adecuados para este tipo de proceso selectivo, motivos históricos, la baja identificación ciudadana en esos territorios con el Estado, o disponer de sectores productivos alternativos. En las entrevistas, se refuerza lo expresado en la encuesta y se incluyen aspectos nuevos, como por ejemplo la relevancia del localismo y falta histórica de movilidad laboral en determinados territorios o la muy sugerente explicación expresada por el profesor CASSESE fundada en la existencia de diferentes subculturas territoriales, con mayor gusto hacia la función pública según su grado de idealismo y pragmatismo. También este profesor relaciona claramente el desarrollo económico territorial con una menor atracción hacia la función pública y matiza que lo importante no es tanto el origen de la alta función pública como que ésta no represente los intereses del país.

Por otra parte, se solicitó la opinión de los ACE respecto de la importancia de la procedencia geográfica de las personas que trabajan en los altos cuerpos de la AGE. Los resultados muestran la división existente en el colectivo respecto del asunto. En el caso que nos ocupa, aunque no son desdeñables las respuestas otorgadas al reconocimiento de la importancia del origen geográfico de los altos funcionarios, ya fuera porque la Administración debe ser un espejo de la sociedad a la que sirve (48\%), o porque puede mejorar las relaciones de cooperación entre el Estado y las CCAA de procedencia (42,2\%), no se destacan en exceso de las que apoyan no darle trascendencia, ya sea por no considerar la procedencia geográfica relevante desde un punto de vista funcional $(39,2 \%)$, ni representativo $(33,3 \%)$. Las respuestas a la pregunta abierta, ya reseñadas anteriormente, muestran en general desconfianza a favorecer cupos, de cualquier tipo, pero particularmente el territorial, en una función pública nacional que debe regirse por los criterios constitucionales ya citados. Lo mismo cabe agregar de la información obtenida de las entrevistas. Aunque algunas personas entrevistadas mostraron un cierto grado de comprensión ante el fenómeno defendiendo una «mirada favorable» hacia el área geográfica de procedencia por parte de los funcionarios en Italia (ENTREV. CASSESE) y que una mejor equi-representación podría redundar en mejorar la sensibilidad ante determinados problemas (ENTREV. FERRANDIZ), en términos generales no se concede al asunto una gran relevancia e incluso se entrevén peligros. De esta manera, se mantiene que más que el aspecto territorial se consideran importantes los aspectos sociales que afectan a la igualdad, tales como el déficit de representación social (infra-representación de clases bajas y medias en la alta función pública y sobre-representación de las bajas en los puestos de menor nivel orgánico y/o funcional (ENTREV. FERRANDIZ). Asimismo, y después de restar sentido a una mejor representación de los originarios de determinados territorios en una función pública que debe regirse por los criterios de igualdad, mérito, capacidad y publicidad, se alerta de que «allí donde se dan discriminaciones positivas a favor de etnias u otras minorías (por ejemplo, en los Balcanes), la OCDE ha verificado un deterioro de la calidad de las decisiones públicas» (ENTREV. SANCHEZ-BEATO).

TABLA 4. ¿ES IMPORTANTE EL ORIGEN GEOGRÁFICO DE LOS ALTOS FUNCIONARIOS DE LA AGE?

\begin{tabular}{lr}
\hline Sí, pues la Administración debe ser un espejo de la sociedad a la que sirve & $48,0 \%$ \\
\hline Sí, pues los empleados públicos son un nexo privilegiado entre la política y la sociedad & $27,5 \%$ \\
\hline $\begin{array}{l}\text { Sí, pues mantienen una especial sensibilidad a la hora de captar las necesidades de la Comunidad } \\
\text { Autónoma de procedencia }\end{array}$ & $33,3 \%$ \\
\hline Sí, pueden mejorar las relaciones de cooperación entre el Estado y su Comunidad Autónoma & $42,2 \%$ \\
\hline No. No considero relevante desde el punto de vista funcional el origen geográfico de los empleados públicos & $39,2 \%$ \\
\hline No. No considero relevante desde el punto de vista representativo el origen geográfico de los empleados públicos & $33,3 \%$ \\
\hline Otro & $5,9 \%$ \\
\hline
\end{tabular}

Fuente: Elaboración propia. 


\subsection{Agenda pendiente para mejorar las capacidades intergubernamentales de la Alta Función Pública}

En los últimos años existe un debate académico y profesional sobre las capacidades y competencias que deben atesorar los altos funcionarios y la función pública directiva para poder responder a los desafíos del entorno y las necesidades de la ciudadanía. Aunque el debate no es nuevo y ha contado con contribuciones sustantivas en otros momentos (PRATS, 2005), en los últimos meses ha tomado vigor con las aportaciones, por ejemplo, de MAPELLI et al. (2017), del CLAD (2016) y de CRESPO (2015a).

Para el caso español, se estima que la situación de la alta función pública del Estado es francamente mejorable en lo relativo a las competencias intergubernamentales o de gestión relacional. Dos ejemplos pueden ilustrar la situación: por una parte, la procedencia geográfica de la alta función pública a través de la de los ACE; por otra, la escasa importancia otorgada a la movilidad inter-administrativa por las autoridades político-administrativas.

En lo que concierne la procedencia geográfica, sorprende, como ya fue señalado, que a pesar de la descentralización política acaecida en España en las últimas décadas, el origen geográfico de los ACE siga procediendo muy mayoritariamente del centro e interior del país, en tanto que las regiones de la periferia (y singularmente Cataluña) arrastran una infrarrepresentación histórica (RUANO, 2014; CRESPO, 2015c). Esta conclusión es relevante pues se parte de la consideración de que la Administración y función pública deben tender a representar la realidad social por motivos de legitimidad institucional y de resultados, lo que afecta necesariamente al sistema selectivo y a los fundamentos y capacidades sobre las que se asienta. Los encuestados consideran adecuados de manera muy mayoritaria $(74,5 \%)$, frente a un escaso porcentaje que defiende lo contrario $(5,9 \%)$, los principios sobre los que reposa el proceso selectivo que les permitió el acceso al cuerpo, resultados coherentes con los publicados recientemente en RUANO (2014). No obstante, también dispone de un apoyo significativo la posibilidad de su enriquecimiento para incluir en mayor medida la diversidad social y favorecer una mejor representación de todas las clases sociales $(38,2 \%)$ así como para incorporar en mayor medida la diversidad territorial $(9,8 \%)$. Entre los cambios a acometer debería considerarse reducir o minorar significativamente el sesgo, fundamentalmente madrileño y mesetario, de la procedencia geográfica de los altos funcionarios, como demuestra el caso de los ACE. Las entrevistas en profundidad, en general, respaldan los resultados de la encuesta, aunque en algunos casos traslucen escepticismo con el conformismo general o incluso proponen reformas de calado para afrontar, entre otros aspectos, todos los sesgos relativos al sistema selectivo, ya radiquen en aspectos socioeconómicos, étnicos, territoriales o discapacidades (ENTREV. ARENILLA). Otros entrevistados plantearon la necesidad de que la AGE no pierda la capilaridad en el territorio desde un punto de vista funcional e institucional (ENTREV. PÉREZ CRUZ) y que la función pública del Estado, con independencia de su origen y donde se encuentre ubicada, sea representativa del conjunto del país (ENTREV. CASSESE).

En el trabajo empírico se obtuvieron propuestas sobre cómo, según los ACE, podría mejorarse la representatividad autonómica en el cuerpo para aquellas CCAA cuyos ciudadanos se encuentran infra-representados en relación al peso poblacional relativo de las mismas en el conjunto del Estado. La respuesta más destacada, con gran diferencia con el resto, fue la que aludía a intervenir en la fase de reclutamiento o atracción de candidatos a las pruebas, haciendo especial énfasis en las CCAA infra-representadas (77,5\%), seguida a gran distancia por la realización de las pruebas de selección de manera territorializada en las diferentes CCAA (22,5\%). Sin embargo, fueron inapreciables las respuestas que indicaron que mejoraría la situación mediante la modificación del contenido de las pruebas de selección, incluyendo más contenidos autonómicos $(5,9 \%)$ o a través de la reserva de cupos o cuotas a las CCAA de acuerdo con su peso demográfico (1\%). Muy significativo, comparativamente, fue el apoyo a «Otros» (23,5\%), lo que obliga a analizar cuidadosamente las respuestas a la pregunta abierta final de este epígrafe, así como las entrevistas realizadas.

TABLA 5. CÓMO MEJORAR LA REPRESENTATIVIDAD EN EL CUERPO DE ACE DE LAS CCAA INFRA-REPRESENTADAS

\begin{tabular}{lr}
\hline POSIBLE SOLUCIÓN & $\%$ \\
\hline Mejorando el reclutamiento (atracción de candidatos) en las CCAA menos representadas & 77,5 \\
\hline Modificando el contenido de las pruebas de selección, incluyendo más contenidos autonómicos & 5,9 \\
\hline Realizando las pruebas de selección en las CCAA & 22,5 \\
\hline Reservando cupos o cuotas a las CCAA y ciudades autónomas de acuerdo con su peso demográfico & 1,0 \\
\hline Otro: & 23,5 \\
\hline
\end{tabular}

Fuente: elaboración propia. 
Las respuestas a la pregunta abierta incluyen nuevas posibilidades. Por una parte, se plantea la posibilidad de anclar a los ACE en la estructura periférica del Estado, con mejores condiciones profesionales y retributivas, lo que permitiría dar a conocer el cuerpo y difundir con un programa específico las funciones que realiza; por otra, la incentivación del conocimiento de otras lenguas españolas además del castellano. Y finalmente, mejorar la difusión de la oferta de empleo público, potenciar el sistema de becas y la comunicación y colaboración con las escuelas de función pública y universidades autonómicas. En esta línea, coincidente con recientes publicaciones que dan cuenta de la preocupación por parte del colectivo de la selección de ACE y de directivos públicos (por ejemplo, MAPELLI et al., 2016), es reveladora la siguiente aportación:

«Resulta urgente mejorar los mecanismos para la difusión de la oferta de empleo público por parte de la DG de Función Pública; convocar y dotar adecuadamente becas para la preparación de los procesos selectivos al A1 para asegurar la representatividad social y geográfica de nuestra función pública superior; explorar todas las posibilidades que brinda la adaptación al espacio europeo de educación superior de nuestras titulaciones y las fórmulas de colaboración con las escuelas de función pública superior de la Administración General del Estado».

Respecto de las entrevistas, se insiste con carácter general en la necesidad de intervenir antes en otros aspectos y no en los criterios que sustentan el proceso selectivo en la Administración. Se enfatiza en la posibilidad de mejorar la fase de reclutamiento, entendida como el proceso de atracción de ciudadanos a los procesos selectivos mediante una mejor difusión de la Oferta de Empleo Público, en el establecimiento de un sistema de becas para ciudadanos con menos recursos que pudieran tener mayor sensibilidad territorial o impartiendo clases de apoyo en algunas CCAA (ENTREV. SANCHEZ-BEATO), lo que tendría un doble impacto: mitigaría el sesgo socioeconómico y también el territorial (ENTREV. FERRANDIZ). En la misma línea, y a pesar de expresar que antes de aplicar cupos territoriales hay que pasar por otras fases, se precisa que, para conseguir una democracia de calidad y una función pública en consonancia, se requiere intervenir en la cultura sociopolítica. En ese sentido, y aunque suele utilizarse la excusa de la tradición, ésta debe cambiar.

«Hay que atacar las diferentes barreras: educativas, socioeconómicas, territoriales y las propias del modelo de selección (las técnicas, que incorporan y perpetúan los sesgos). Para esto último, en el tema territorial, planteo realizar alianzas con universidades y escuelas territoriales, ofertar prácticas en la AGE relacionadas con esos centros de formación y para ciudadanos de esos territorios, proporcionar ayudas, etc. También es necesario cambiar la filosofía del modelo. En un modelo de competencias debe cambiar la manera de ver y gestionar el talento y las capacidades» (ENTREV. ARENILLA).

Las opiniones contrarias a los cupos territoriales son frecuentes e incluso amplían el foco a otras posibles discriminaciones positivas (de género, discapacidad, étnicas...) en algunos casos. Por ejemplo, en ENTREV. BAENA se expresa prevención ante un inmoderado carácter representativo socialmente de la función pública. El profesor BAENA piensa que ese criterio, venido de EEUU, fracasó pues no estaba suficientemente justificado y podría atentar contra el principio de igualdad de los ciudadanos. Una cosa es que convenga favorecer la posibilidad de que cualquier ciudadano llegue a formar parte de un alto cuerpo, y otra que se esté dificultando o violentando la posibilidad de que ello suceda. A nadie se le impide, lo que hace falta es superar las pruebas, aunque se puede mejorar el reclutamiento y la preparación de las pruebas mediante centros públicos, privados y ayudas. Y es que para el profesor BAENA la fase de reclutamiento es tan importante o más que la de selección. Por el contrario, otro entrevistado (ENTREV. CASSESE), muestra una gran preocupación porque la función pública sea adecuadamente representativa de la sociedad, alertando contra lo que denomina «veto representativo». Además, en tanto juez constitucional italiano, ve posible incluir «acciones activas» con miras a aminorar la distancia entre la igualdad formal y sustancial del acceso de los ciudadanos a los empleos públicos.

A pesar de los reparos para favorecer una presencia más proporcional respecto de la importancia demográfica de las diferentes CCAA, cabe reseñar que a nivel internacional (en las organizaciones internacionales), sí se suelen tener en cuenta las cuotas nacionales. La propia Unión Europea, con una función pública inspirada en las de los países miembros (particularmente de la estatutaria francesa, con previsión de carrera) no ha podido impedir la existencia del «equilibrio geográfico», criterio derivado de la normativa comunitaria (los funcionarios europeos serán «seleccionados según una base geográfica lo más amplia posible entre los nacionales de los Estados miembros de las Comunidades») y que asigna cuotas en la función pública comunitaria a los distintos países. Como establece FUENTETAJA (2013), a pesar de que ello puede «nacionalizar» segmentos de la acción administrativa comunitaria, también incorpora aspectos positivos: según algunos autores es un factor de integración que mejora la cooperación entre los países y que asegura 
una mejor armonía entre la acción de la UE y la de los Estados miembros. Además, se afirma que permite comprender mejor las mentalidades nacionales y garantizar la calidad de la actuación. Pero, por otra parte, se señalan disfunciones: relativización de los principios de mérito, capacidad e igualdad; dificultad de ajustes y rigidez en la movilidad horizontal; reducción (o ampliación injustificada) de las posibilidades de carrera de los funcionarios; dificultades de reajustes de personal por necesidades del servicio; o dificultades de remoción por motivos disciplinarios.

Establecido lo anterior, cabe reseñar la percepción de los ACE respecto de las competencias requeridas para interactuar en el contexto intergubernamental español. Así, sin menosprecio de los aspectos jurídicos o técnicos, los ACE otorgan gran relevancia a los aspectos éticos $(96,3 \%)$, los relacionados con aspectos interpersonales e interorganizativos $(94,1 \%)$, las habilidades personales y de comunicación $(87,3 \%)$ y las que facilitan trabajar en entornos multidisciplinares, con diversidad política o cultural, en equipo y con orientación a resultados. Sorprende sin embargo la menor importancia otorgada a los aspectos lingüísticos $(57,8 \%)$ en una función pública con un fuerte contenido relacional interior y exterior. En las entrevistas se destacó la necesidad de activar las competencias que ponen al ciudadano en el centro de la acción administrativa y, muy especialmente, las ligadas a la apertura, la orientación al ciudadano y la proximidad. Además, se estima que es clave analizar y mejorar el perfil de la alta función pública o estratégica para que, sin perjuicio de las competencias técnicas e institucionales, hallen acomodo unas nuevas capacidades, entre las que destacan las que posibilitan el accionar administrativo en el marco confuso y complejo descrito, a saber: sensibilidad y apertura a nuevas culturas (incluyendo mayor grado de conocimientos de lenguas), diseño, gestión y participación en redes, predisposición al trabajo colectivo y la necesidad de gestionar el conflicto (PRATS, 2005: 13). Ello implica que, además de los aspectos técnicos e institucionales de carácter general, deban incluirse otros que faciliten la interacción multinivel: las técnicas de negociación más relevantes, factores de multiculturalidad y multinacionalidad (necesarios para poder interaccionar y encontrar consensos en entornos inciertos, de participación fluida, y donde entran en juego intereses de varios niveles de gobierno, y extra-administrativos), y, finalmente, habida cuenta de la importancia que adquieren la gestión en red y las relaciones informales en nuestros días, la inmersión en valores que favorezcan el consenso y la cooperación, junto a unos estándares básicos de aptitudes sociointegrativas (CRESPO, 2015a). Es decir, impulsar y estimular aquellos comportamientos incluidos en las competencias asociadas a la gestión de vínculos y conducción del cambio (en la terminología expresada en CLAD 2016), pues son las que mejor se adaptan a la articulación intergubernamental.

GRÁFICO 2. LA MOVILIDAD INTER-ADMINISTRATIVA EN ESPAÑA

\section{\%EN DESACUERDO/TOTALMENTE EN DESACUERDO}

\% DE ACUERDO/TOTALMENTE DE ACUERDO

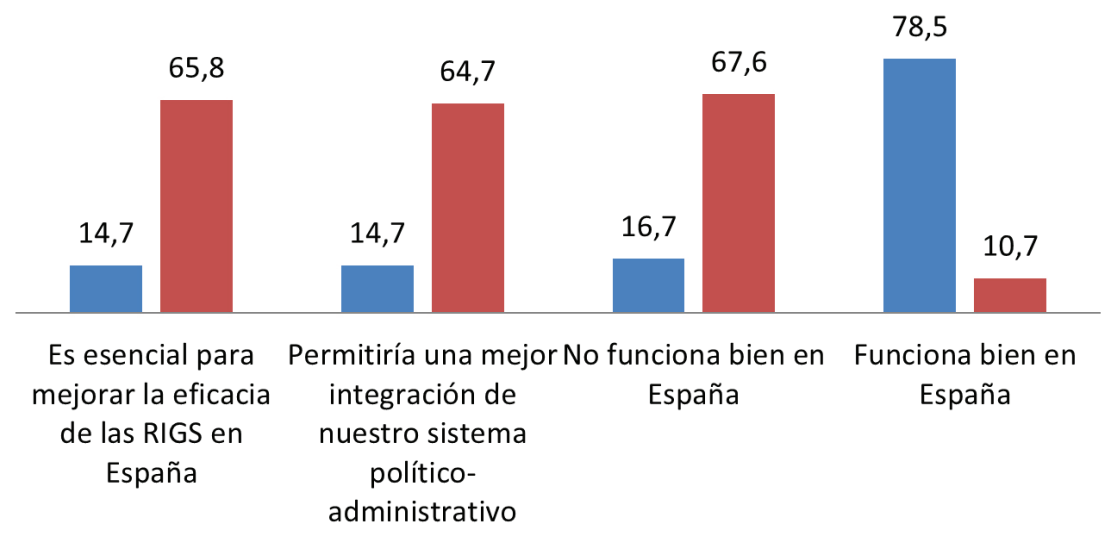

Fuente: elaboración propia.

Establecido esto, el trabajo empírico muestra que la situación es francamente mejorable en lo relativo a las competencias intergubernamentales, o de gestión relacional, como demuestran, a modo de ejemplo, la procedencia geográfica de la alta función pública a través de la de los ACE, o la escasa importancia otorgada 
a la movilidad inter-administrativa por las autoridades político-administrativas para un funcionamiento más integrado del Estado. Respecto de esto último, y en consonancia con la literatura internacional (STEIN, 1984 o WEIDNER, 1960), los encuestados expresaron su conformidad con que dicha movilidad es esencial para mejorar la eficacia de las RIGS en España y que la misma permitiría una mejor integración de nuestro sistema político-administrativo. Sin embargo, el $78,5 \%$ de ellos expresa desacuerdo con su funcionamiento y solo un 3,9\% indica que no debería cambiar. También se señalaron formas de mejorar la movilidad: el intercambio temporal de funcionarios, favorecer la ocupación de puestos apropiados en las diferentes Administraciones a originarios de otras, facilitar la realización de prácticas formativas y eliminar obstáculos legales.

\section{CONCLUSIONES}

Los estudios de carácter empírico sobre la función pública, desde una perspectiva intergubernamental, son escasos y fragmentarios, como demuestra el estado de la disciplina a nivel internacional y muy singularmente en el marco español.

Sin embargo, se estima que el estudio de la alta función pública permite concretar claves para comprender el sistema de dominación establecido en los países democráticos (en este caso, España) al asegurar funciones de mediación y relación entre la ciudadanía, en sus diferentes expresiones, y el poder político e institucional.

En efecto, la relevancia que se otorga a la alta función pública, en los estudios sobre las élites, plantea que dispone de una posición superior a la de mera administración pública ejecutora y que, por lo tanto, el origen y la forma de confección de las élites administrativas exigen una mayor reflexión por su influencia sobre el poder democrático. Y ello por los siguientes considerandos: sus importantes funciones en términos de formulación de políticas conformadoras y con impacto multinivel, así como las correspondientes en lo relativo a la aplicación y evaluación de las mismas; por su cercanía y proximidad con el entorno de decisión político, al que proporciona consejo y orientación; y, finalmente, por el solapamiento comprobado empíricamente entre los dos colectivos, político y burócrata, intercambiando constantemente recursos y posiciones (BAENA, 1999; ARENILLA, 2016), con independencia del mantra de que los servidores públicos profesionales deben centrarse en el objeto de su conocimiento técnico y en garantizar el cumplimiento de las normas.

En ese contexto, es imprescindible integrar diversidad a la composición de la alta función pública, mitigando los sesgos de todo tipo, pero muy especialmente los de carácter socioeconómico e intergubernamentales. Hemos dedicado este artículo a reflexionar sobre estos últimos, mostrando la importancia de buscar un adecuado equilibrio en la alta función pública del Estado español entre los altos funcionarios procedentes de las diferentes CCAA, así como mejorar la integración del sistema a través de una movilidad inter-administrativa redescubierta e impulsada. Esto último, que ya había sido evidenciado en los estudios empíricos estadounidenses referenciados por WEIDNER (1960) o McCULLOCH (1965), y respaldado por STEIN (1984), se ha visto confirmado por nuestro trabajo empírico.

Otros resultados procedentes del trabajo empírico señalan que los roles desempeñados en su ejercicio profesional por los altos funcionarios generalistas estudiados son fundamentalmente los de expertos, garantes de la imparcialidad del Estado, técnicos legales y formuladores de nuevos programas, junto a otros de carácter intergubernamental: el de facilitadores y mediadores. Asimismo, los ACE concretaron en que ámbitos radica su labor intergubernamental, destacando la participación en la elaboración, implantación y evaluación de normativa de impacto multinivel, la redacción de respuestas parlamentarias y asesoramiento a órganos políticos y, en especial, mediante la participación en órganos de cooperación entre niveles de gobierno (conferencias sectoriales y bilaterales). Finalmente, se sugirió el interés de afrontar algunos de los sesgos representativos (como el territorial) a través de la mejora del proceso selectivo potenciando la atracción de candidatos de determinadas CCAA, y en menor medida facilitando la realización de ejercicios en las mismas; al mismo tiempo se constató el escasísimo respaldo otorgado a la modificación de los temarios incluyendo más contenidos autonómicos o a la reserva de cupos de plazas para los originarios de las CCAA de acuerdo con su peso demográfico.

Sin duda, la consideración y eventual mejora de los aspectos señalados redundará en un papel integrador, cohesionador y lubricante para las principales políticas públicas multinivel por parte de la alta función pública, tal como ha señalado PARRY (2004 y 2012), rol que contribuirá a mejorar los desafíos de integración territorial en un Estado tan diverso y plural como es el español. 


\section{BIBLIOGRAFÍA}

ABERBACH, J.; PUTNAM, R.; ROCKMAN, B., 1981, Bureaucrats and Politicians in western democracies. Cambridge, Harvard University Press.

AGRANOFF, R., 2001, "Managing within the matrix: do collaborative intergovernmental relations exist?", en Publius: The Journal of Federalism, 31: 2 (Spring), págs. 31-56.

AGRANOFF, R.; BAÑÓN, R., 1998, El Estado de las Autonomías: ¿hacia un nuevo federalismo?, Gobierno vasco, Instituto Vasco de Administración Pública.

AGRANOFF, R., 1991, "Marcos para el análisis comparado de las relaciones intergubernamentales". Papeles de trabajo del Instituto Ortega y Gasset, núm. 291. Serie Gobierno y Administración. Madrid.

AGRANOFF, R.; LINDSAY, V., 1983, "Intergovernmental management: perspectives from human services problema solving at the local level", en Public Administration Review, may/june.

ALONSO, J. M.; CLIFTON, J., 2013, "Public sector reform in Spain: Views and experiences from senior executives. Country report as part of the COCOPS Research project". Accesible en: http://www.cocops.eu/wp-content/ uploads/2013/06/Spain_WP3-Country-Report.pdf (fecha de acceso: 30 de marzo de 2018).

ALVÁREZ ALVÁREZ, J., 1980: El origen geográfico de los funcionarios españoles. Instituto Nacional de Administración Pública.

ANDERSON, W., 1960: Intergovernmental relations in review. Minneapolis, University of Minnesota Press.

ARBOS, X. (coord.), COLINO, C.; GARCÍA, M. J.; PARRADO, S., 2009: Las relaciones intergubernamentales en el Estado autonómico. La posición de los actores. Barcelona, Institut d’Estudis Autonómics.

ARENILLA SÁEZ, M., 2016: "Naturaleza política y función social de la Administración Pública". Laudatio pronunciada en el acto de investidura de D. Mariano Baena del Alcázar como doctor honoris causa por la Universidad Rey Juan Carlos de Madrid, el día 9 de septiembre de 2016. Accesible en http://laadministracionaldia.inap.es/noticia. asp?id=1506602 (fecha de acceso: 20 de septiembre de 2016).

ARENILLA SÁEZ, M., 2014: "Presentación”, en RUANO DE LA FUENTE, J. M. (dir.), CRESPO GONZÁLEZ, J. y POLO VILLAR, C., Los funcionarios ante el espejo. Análisis del ciclo de selección de personal de la Administración General del Estado. Madrid, INAP.

ARENILLA SÁEZ, M., 2011, "Marco teórico actual de la Administración Pública", en Manuel ARENILLA (dir.), Crisis y Reforma de la Administración Pública. La Coruña, Editorial Netbiblo, págs. 1-137.

ARENILLA SÁEZ, M., 2010: “Administración Pública y Ciencia de la Administración”, en M. ARENILLA (coord.) La Administración pública entre dos siglos. Libro Homenaje a Mariano Baena del Alcázar, Madrid: INAP, págs. 39-68.

BAENA DEL ALCÁZAR, M., 1988: Curso de la Ciencia de la Administración, vol. I, 2. ed. Madrid, Tecnos.

BAENA DEL ALCÁZAR, M.; GARRIDO, L.; PIZARRO, N., 1984, "La élite española y la presencia en ellas de los burócratas", en Documentación Administrativa, núm. 200, págs 73-131.

BAÑÓN, R.; TAMAYO, M., 1998, "Las relaciones intergubernamentales en España: el nuevo papel de la Administración central en el modelo de relaciones intergubernamentales”, en AGRANOFF, R.; BAÑÓN, R., 1998, El Estado de las Autonomías: ¿hacia un nuevo federalismo?, Gobierno vasco, Instituto Vasco de Administración Pública, págs. 105-159.

BAÑÓN, R.; CARRILLO, E., 1997, La nueva Administración Pública. Madrid, Alianza Editorial.

BAÑÓN, R. et al., 1995, "Sistema de Relaciones intergubernamentales y legitimidad de la acción pública en el Estado de las Autonomías", en Papeles de Trabajo (Gobierno y Administración Pública) de la Fundación Ortega y Gasset, núm. AP, 20-XII-95.

BELTRÁN VILLALBA, M., 1977, La elite burocrática española, Madrid, Ariel-Fundación Juan March.

BENSON, G. C.,1965, "Trends in Intergovernmental Relations", en SELLIN, T.; WOLFGANG, M. (eds.), 1965, Intergovernmental Relations in The United States. The annals of the American Academy of Political and Social Science, Philadelphia, págs. 1-9. https://doi.org/10.1177/000271626535900102.

CARABAÑA, J. y LAMO DE ESPINOSA, E., 2008, "La élite burocrática y la movilidad social", en MEIL, G. y TORRES, C. (coords.), 2008, Sociología y realidad social: libro homenaje a Miguel Beltrán Villalva, págs. 391-424.

$\mathrm{CHO}, \mathrm{C} .-\mathrm{L} ., 2007$, "Understandig intergovernmental coercion: Explaining American State Administrators' perceptions of national regulatory influences", en International Review of Public Administration, vol. 12, núm. 1, págs. 51-62.

CLAD, 2016, Guía referencial latinoamericana de competencias laborales en el sector público. Aprobada por la XVII Conferencia Iberoamericana de Ministras y Ministros de Administración Pública y Reforma del Estado. Bogotá, Colombia, 7 y 8 de julio de 2016.

COLINO, C., 2015, "National patterns of public administration and governance", in MAGONE, J. (ed.), Routledge Handbook of European Politics. London, Routledge, págs. 611-639.

COLINO, C., 2012: "Las relaciones intergubernamentales en España: un estado de la cuestión y algunas consideraciones en perspectiva comparada”, en COLINO, C.; FERRÍN, M.; LEÓN, S., 2012: La práctica de la cooperación intergubernamental en España. Madrid, Centro de Estudios Políticos y Constitucionales, págs. 17-54.

CRESPO GONZÁLEZ, J., 2017, "Coordinación intergubernamental en España vista por la alta función pública del Estado”, en Política y Sociedad, vol. 54, núm. 2, págs. 469-496. https://doi.org/10.5209/poso.52199. 
CRESPO GONZÁLEZ, J., 2015a, "La función pública ante un escenario de gobernanza multinivel: crisis y reinvención”, en CRESPO GONZÁLEZ, J. (dir.), Crisis y reinvención de la función pública ante un escenario de gobernanza multinivel. Madrid, Instituto Nacional de Administración Pública, págs. 39-62.

CRESPO GONZÁLEZ, J., 2015b, "La centralidad del estudio de la función pública en un escenario de gobernanza multinivel en crisis", en CRESPO GONZÁLEZ, J. (dir.), Crisis y reinvención de la función pública ante un escenario de gobernanza multinivel. Madrid, Instituto Nacional de Administración Pública, págs. 31-38.

CRESPO GONZÁLEZ, J., 2015c, "Pluralismo geográfico del origen de los altos funcionarios de la Administración general del Estado", en GONZÁLEZ GARCÍA, E. et al. (coords.), Mundos emergentes: cambios, conflictos y expectativas. Toledo, Asociación Castellano-Manchega de Sociología, págs. 766-779.

CRESPO GONZÁLEZ, J., 2004: "El gobierno multinivel como herramienta analítica de las políticas europeas" en RUANO DE LA FUENTE, J. M. (coord.) 2004: Política europea y gestión multinivel. Oviedo, Septem ediciones. págs. 15-46.

DE LA PEÑA, A.; ELIZONDO, A.; JUARISTI, F.; MOKOROA, J. L.; MONDRAGÓN, J., 2015, "Las conferencias sectoriales (2001-2012): Dinámica de funcionamiento y valores y percepciones de los agentes políticos y técnicos", en Gestión y Análisis de Políticas Públicas, nueva época, núm. 14. págs. 23-41. https://doi. org/10.24965/gapp.v0i14.10288.

DEMMKE, C. y MOILANEN, T., 2012: The future of public employment in central public administration. Maastricht, EIPA.

FUENTETAJA, J. M., 2013: Pasado, presente y futuro de la función pública. Entre la politización y la patrimonialización. Pamplona: Editorial Aranzadi.

FUENTETAJA, J. M., 2004: "La reforma de la función pública europea", Revista de Derecho Comunitario Europeo, Año 8, 19, págs. 751-875.

GARCÍA MORALES, M. J., 2006, "Las relaciones intergubernamentales en el Estado autonómico: estado de la cuestión y problemas pendientes", en GARCÍA MORALES, M. J., et al., 2006, Las relaciones intergubernamentales en el Estado autonómico. Madrid, Centro de Estudios Políticos y Constitucionales, págs. 9-72.

KUPERUS, H. y RODE, A., 2016, Top public managers in Europe. Management and employment in Central Public

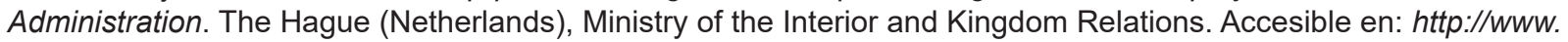
eupan.eu/files/repository/20170206084104_TopPublicManagersinEuropemainreport.pdf (fecha de acceso: 30 de marzo de 2018).

LINS DE LESSA, F., 2011, Acceso igualitario a la función pública, Lisboa, Editorial Juruá.

LINZ, J.; DE MIGUEL, A., 1968, "La élite funcionarial española ante la reforma administrativa”, en AA.VV, 1968, Sociología de la administración pública española, Madrid, Centro de Estudios Sociales de la Santa Cruz del Valle de los Caídos.

LONGO, F., 2001: La reforma del servicio civil en las democracias avanzadas. Mérito con flexibilidad. Washington, Banco Interamericano de Desarrollo.

MAPELLI MARCHENA, C. et al., 2017, Nuevos tiempos para la función pública. Propuestas para atraer y desarrollar el talento en la Administración General del Estado. Madrid, INAP.

MAPELLI MARCHENA, C. et al., 2016, "Función pública: reformas pendientes", en Zoom Político, documento núm. 29. Laboratorio de alternativas.

MCCULLOCH, R. W., 1965, "Intergovernmental relations as seen by public officials", en SELLIN, T.; WOLFGANG, M. (eds.), 1965, Intergovernmental Relations in The United States. The annals of the American Academy of Political and Social Science. Special editor of this volume: Harry Reynolds Jr., Philadelphia, págs. 127-136. https://doi. org/10.1177/000271626535900114.

OCDE 2014: España: de la reforma de la Administración a la mejora continua. Informe de la OCDE sobre gobernanza pública en España. Madrid, Instituto Nacional de Administración Pública-Organización para la Cooperación y Desarrollo Económico.

OCDE 2011: Public Servants as Partners for Growth: Toward a Stronger, Leaner and More Equitable Workforce. Paris.

OLÍAS DE LIMA, B. (coord.), 1995, La gestión de recursos humanos en las Administraciones Públicas, Madrid, Editorial Complutense.

PARRADO DIEZ, S., 1996, Las élites de la Administración estatal (1982-1991): estudio y pautas de reclutamiento. Sevilla, Instituto Andaluz de Administración Pública.

PARRY, R., 2012, "The civil service and Intergovernmental Relations in the Post-devolution UK", en The British Journal of Politics and International Relations, vol. 14, págs. 285-302.

PARRY, R., 2004, "The civil service and intergovernmental relations", Public Policy and Administration, 19: 2, 50-63. https://doi.org/10.1177/095207670401900207.

PASTOR ALBALADEJO, G., 2015, "El Instituto Nacional de Administración Pública como institución generadora de conocimiento transformador en la función pública española”, en CRESPO GONZÁLEZ, J. (dir.), Crisis y reinvención de la función pública en un escenario de gobernanza multinivel. Madrid, INAP, 199-229.

PETERS, B. Guy, 2008, "The civil service and governing: reclaiming the center". International Review of Public Administration, 13: 2, págs. 1-12. https://doi.org/10.1080/12294659.2008.10805118.

PRATS I CATALÁ, J., 2005: De la burocracia al management, del management a la gobernanza. Las transformaciones de las Administraciones públicas de nuestro tiempo. Madrid, INAP-IIGC. Este trabajo fue publicado previamente 
en "Las transformaciones de las Administraciones Públicas de nuestro tiempo", Documentos de Trabajo, núm. 10 del IIG de Catalunya.

ROMÁN MASEDO, L., 1997, Funcionarios y función pública en la transición española. Madrid, Centro de Estudios Políticos y Constitucionales.

ROMÁN RIECHMANN, C., SANCHEZ MORÓN, M.; VELÁZQUEZ LÓPEZ, F. J., 2004, "Líneas de reforma del empleo público", en AA.VV, Estudios para la reforma de la función pública, Madrid, Instituto Nacional de Administración Pública, págs. 479-516.

ROSENBLATT, F.; TORO MAUREIRA, S., 2015, "La arquitectura de la cooperación: una propuesta teórica sobre la resiliencia institucional”, en Política y Gobierno, vol. XXII, núm. 2, págs. 255-281.

RUANO DE LA FUENTE, J. M., 2016: "Functional Federalism in a Complex State. The case of Spain", en RUANO DE LA FUENTE, J. M.; PROFIROIU, M. (eds.), 2016, Handbook on Decentralisation in Europe, London, Palgrave. https://doi.org/10.1007/978-3-319-32437-1 4.

RUANO DE LA FUENTE, J. M., (dir.), CRESPO GONZÁLEZ, J., y POLO VILLAR, C., 2014, Los funcionarios ante el espejo. Análisis del ciclo de selección de personal de la Administración General del Estado. Madrid, INAP.

SANIGER MARTINEZ, N. y ZAFRA ESCRIBANO, J. M. "La necesaria reforma de los mecanismos de cooperación en el sistema de relaciones intergubernamentales del Estado de las Autonomías y el nuevo papel del Gobierno Local", en ARENILLA SÁEZ, M., (coord.), 2010, La Administración Publica entre dos siglos. Homenaje a Mariano Baena del Alcázar. Madrid, INAP.

SANIGER MARTINEZ, N., 2005 "Las deficiencias del sistema de relaciones intergubernamentales en el Estado de las Autonomías", en Libro Homenaje a José Cazorla Pérez. Madrid, CIS, págs. 427-455.

SELLIN, T.; WOLFGANG, M. (eds.), 1965, Intergovernmental Relations in The United States. The annals of the American Academy of Political and Social Science. Special editor of this volume: Harry Reynolds Jr. Philadelphia.

SILICANI, J.-L., 2008, Livre Blanc sur l'Avenir de la Fonction Publique, París: Ministère du Budget, des Comptes et de la Fonction Publique.

STEERING GROUP 2008: Normington Report: Senior Civil Service Workforce and Reward Strategy. London.

STEIN, J. W., 1984, "Improving intergovernmental relations through public service careerism", en Journal of Policy Analysis and Management, spring 1984, 3: 3, págs. 453-457. https://doi.org/10.1002/pam.4050030312.

VERKUIL, P., 2015, "Deprofessionalizing State Governments: The rise of Public Art-Will Employment", en Public Administration Review, vol. 75, núm. 2, págs. 188-189.

VILLORIA MENDIETA, M. y DEL PINO MATUTE, E., 2009: Dirección y gestión de recursos humanos en las Administraciones Públicas, Madrid, Tecnos.

WEIDNER, E. W., 1960, Intergovernmental Relations as seen by public officials. Connecticut, Greenwook press, publishers. Se ha utilizado la reimpresión de 1974.

WRIGHT, D., 1997, Para entender las relaciones intergubernamentales. México, Fondo de cultura económica. Edición inglesa de 1988: Understanding intergovernmental relations. Belmont, Brooks-Cole.

WRIGHT, D., 1978, "Del federalismo a las Relaciones Intergubernamentales en los Estados Unidos de América. Una nueva perspectiva de la actuación recíproca entre el gobierno nacional, estatal y local", en Revista de Estudios Políticos, núm. 6, págs. 5-28. 